\title{
A Novel Automated Shoeprint Matching Technique for use as Forensic Evidence in Criminal Investigation
}

\author{
Vaishali.R.Hire \\ Assistant Professor \\ S.S.V.P.S.COE, DHULE \\ Maharashtra (India)
}

\author{
Faizal.I.Shaikh \\ Professor \\ JNEC, AURANGABAD \\ Maharashtra (India) \\ Medha V. Joshi \\ Assistant Professor S.S.V.P.S.COE, DHULE \\ Maharashtra (India)
}

\author{
Jayashree B. Jadhav \\ Assistant Professor \\ S.S.V.P.S.COE, DHULE \\ Maharashtra (India)
}

\begin{abstract}
This work presents a technique in which a database of outsole pattern of shoeprint images has been automatically sorted against a query shoeprint image. Shoe marks found at the place of crime are used to provide valuable forensic evidence. This system presents a technique for rotation and intensity invariant automatic shoeprint matching so that the spatial positioning of the reference shoeprint image does not have to correspond with the spatial positioning of the shoeprint images of the database. Gabor transform has been used to extract multi resolution features of a shoeprint. Radon transform has been used to estimate the rotation of the shoeprint image and is compensated by rotating the features in opposite direction. Shoeprint database has been generated by inviting participants to tread on an inkpad and then stamp on a piece of paper. Template query images have been compared using Euclidean distance classifier which has been used to find a suitable match. The performance of the proposed algorithm has been evaluated in terms of percentage accuracy for four different matching methods. This technique performs better compared to results obtained using power spectral density features for full print images with the rotation, intensity and mixed attacks.
\end{abstract}

\section{General Terms}

Image processing, Forensic application.

\section{Keywords}

Shoe print processing, Radon transform, Image processing, Gabor transform, Gabor feature map, orientation field.

\section{INTRODUCTION}

Recent developments in forensic science have resulted in large numbers of scene of crime images being collected for recording and analysis. These have recently been of great interest to police and forensic scientists as footwear evidence is now treated in same manner as fingerprint and DNA evidence. Shoe marks may help to establish that a number of separate crime incidents probably involve the same individual. So it would be combined with other evidence to make a convincing case to outline to a jury or help detectives link different cases or narrow down their lines of inquiry. The aim of this study was to develop an automated system to aid forensic scientist in rapidly identifying the model of a shoe from an image of a shoeprint

Invariance, rotate the computed features by the amount of estimated orientation.5)Match the rotated features with the
Forensic science refers to the application of principles and methods of science and medicine to legal questions of a criminal or civil nature. Shoe impressions are the most common clues along with tier print and some of the physiological biometric evidences found at the place of crime. These impressions carry substantial information and are useful in linking the crime scenes and trap the criminals. Shoe marks-the marks made by the outside surface of the sole of a shoe-are distinctive patterns that are often found at crime scenes. Shoe marks are common at crime scenes and are believed to be present more frequently than fingerprints [1].In [2], a study of several jurisdictions in Switzerland revealed that 35 percent of crime scenes had shoeprints usable in forensic science, while in [3], Girod found that 30 percent of all burglaries provide usable shoeprints.

In forensic laboratories images of shoe marks are compared with shoe impressions of known shoe samples. Forensic analysis requires comparison of this image against specific databases. Matching of shoe mark images to database is done using semiautomatic methods [4] [5] in which operator uses basic or geometrical shapes to find a model of shoeprint pattern Disadvantages of this method are i) Because of more intricate outsole patterns, it becomes difficult to describe it with few basic shapes. ii) Different geometric patterns may be selected while coding the same shoeprint which creates difficulties to determine a match for shoeprint. Now days, shoeprint recognition is done fully automatic [7-10] using Discrete Fourier transform (DFT) [6] and Gabor transform. But with DFT, there is disadvantages like- i) it requires image size to be power of two ii) this method is completely nonlocal. iii) In this method a problem with boundary condition occurs.

\section{PROPOSED}

SHOEPRINT

\section{MATCHING TECHNIQUE}

In this technique which uses Gabor transform, following steps are used. 1) Compute the Gabor feature map corresponding to orientations of full images. This is known as enrollment.2) Estimate a direction (orientation) of query shoeprint image using Radon transform.3) Compute the feature map of query image.4) For obtaining rotational

Gabor feature maps of various shoeprint images in the database which is known as pattern matching. The proposed 
shoeprint matching technique using Radon and Gabor transform is shown in figure1.

\subsection{Normalization}

In image processing, normalization is a process that changes the range of pixel intensity values. The normalization process will produce shoeprints regions, which have the same constant dimensions, so that two photographs of the same shoeprints under different conditions will have characteristic features at the same spatial location. In shoe print matching, due to the freedom offered to the subjects, naturally the rotation,

\subsection{Radon Transform for Estimating Shoeprint Rotation}

The Radon transform is able to transform two dimensional images with lines into a domain of possible line parameters, where each line in the image will give a peak positioned at the corresponding line parameters. The orientation image represents an intrinsic property of the shoeprint images. In this work an automatic orientation correction is proposed before the images are trained. The Radon transform can be used to detect linear trends in images. The principle direction of the texture in shoeprint image can be roughly defined as the The Radon transform along this direction usually has larger variations. Therefore the variance of the projections at this direction is locally maximum. The line property of Radon transform is an important property utilized for rotation estimation of a shoeprint. Radon transform assumes a function that contains line, which is modeled with a delta function.

$$
g(x, y)=\delta\left(y-p^{*} x-\tau^{*}\right)
$$

Hence, the function has non-zero values only if $(x, y)$ lies on the line with certain fixed parameters $\left(p^{*}, \tau^{*}\right)$.

The amount of rotation of a query shoeprint image is estimated before computing the feature. The principal direction of the texture in a shoeprint image can be defined in terms of directional edges. The Radon transform along this direction exhibits larger variations. Therefore the variance of the projections at this direction is locally maximum [11]. The orientation of texture in a shoeprint is given by intensity variation (by means of contrast variation in the gray scale) or both have been introduced randomly in the test shoeprint images. The intensity variations introduced are because of variations in the pressure exerted by the subject and non-uniformity in spreading of ink during image acquisition process. Intensity variations in the shoeprint image affects on its average power. But the appearance of directional patterns in a shoeprint does not get affected significantly. For making the algorithm intensity invariant, the test shoeprint images have been normalized with respect to their mean in the beginning of the algorithm.

$$
\alpha=\arg (\underbrace{\min }_{\theta} \frac{d^{2} \sigma_{\theta}^{2}}{d \theta^{2}})
$$

where, $\sigma_{\theta}^{2}$ is the variance of the projection at an angle $\theta$.

\subsection{Shoeprint Feature Extraction Using Gabor Transform}

Gabor transform has been used in texture analysis for their excellent properties such as spatial frequency localization. For this the family of 2 -D Gabor filters was used b Daugman [12] and then was further mathematically explained in reference.

Now for feature extraction, the shoeprint image has been considered as a texture image. [13] The local and global texture features of shoeprint images could be extracted by properly selecting the frequency, bandwidth and orientation of Gabor filter. For this an even symmetric Gabor filter in a spatial domain has a form of

$$
g_{(x, y, T, \phi)}=\exp \left\{-\frac{1}{2}\left[\frac{x_{\phi}^{2}}{\delta_{x}^{2}}+\frac{y_{\phi}^{2}}{\delta_{y}^{2}}\right]\right\} \cos \frac{2 \prod x_{\phi}}{T}
$$

where $\phi$ is the orientation of the derived Gabor filter, and $\mathrm{T}$ is

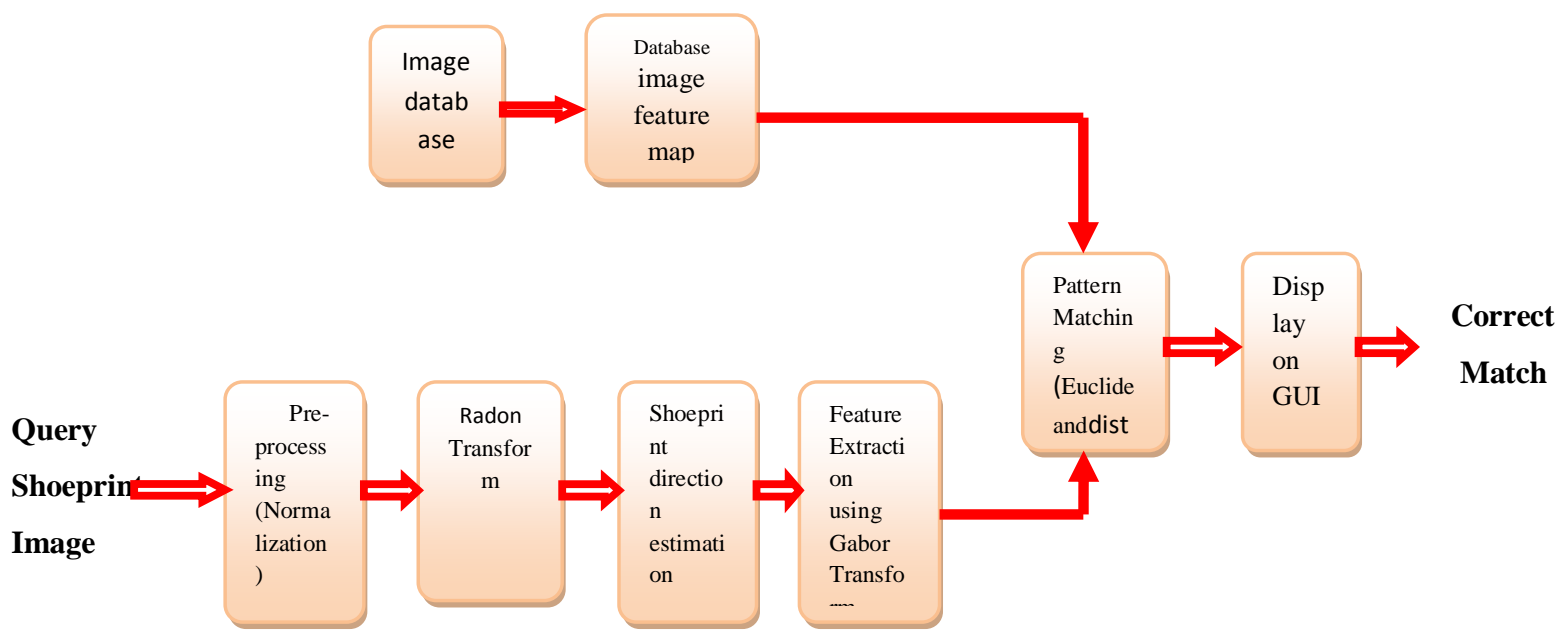

Fig 1: Shoeprint matching using Gabor transform

$\delta \mathrm{x}$ and $\delta \mathrm{y}$ are standard deviations of Gaussian envelop along $\mathrm{x}$ and y axes respectively.

$$
\mathrm{x} \phi=\mathrm{x} \cos \phi+\mathrm{y} \sin \phi
$$$$
\mathrm{y} \phi=\mathrm{x} \sin \phi+\mathrm{y} \cos \phi
$$ 
Steps for shoeprint feature extraction using Gabor filter.

Step1: First capture a shoeprint image and resize to make the algorithm generalized. Shoeprint images of variable size 3008 x $1088,3040 \times 1248$ and $2800 \times 1280$ are resized into 256 x 128 pixels.

Step2: Select even symmetric Gabor parameters which are applied to shoeprint image.

The parameters $\delta \mathrm{x}$ and $\delta \mathrm{y}$ determines the bandwidth of Gabor filter. The higher values of $\delta x$ and $\delta y$ are more robust to noise, but it is not able to capture edge information at fine level. Smaller values of $\delta x$ and $\delta y$ are less robust to noise, but it capture edge information at fine level. The second parameter, filter frequency $f$ is average edge frequency and is set to reciprocal of the average inter-edge distance. If $f$ is selected too small, nearby edges are merged into one. If $f$ is too large spurious edges are created in the filtered image. So select the values of $f, \delta \mathrm{x}$ and $\delta \mathrm{y}$ appropriate. Gabor filter is used in eight directions $\left(0^{0}, 22.5^{0}, 45^{0}, 67.5^{0}, 90^{0}, 112.5^{0}, 135^{0}\right.$, and $157.5^{\circ}$ ).Using these parameters the Gabor filter representations are calculated and stared in lookup table known as the Gabor filter bank.

Step: 3 Feature map computation, eight filtered images are obtained by convolving a shoeprint image with the bank of Gabor filter in eight orientations $\left(0^{0}, 22.5^{0}, 45^{0}, 67.5^{0}, 90^{0}\right.$, $112^{0}, 135^{0}$, and $157.5^{0}$ ). Gabor filter captures the directional textural information in a shoeprint. And energy distribution in Gabor filtered images is used to compute the variance feature map. The energy in a filtered image at an angle $\theta$ is given by,

$E \theta=\frac{1}{\text { number of pixels }} \sum(\text { pixel int ensity })^{2}$

To represent shoeprint texture information, the filtered shoeprint image divided into blocks of size $16 \times 16$ pixels. The average variance of the pixel intensities is used as feature map.

The average variance is defined as,

$\mathfrak{R} \phi=\left\{\sigma_{\phi}^{2}(\mathrm{i}, \mathrm{j})\right\}$

where, $\sigma_{\theta}^{2}(i, j)=\frac{1}{i \times j} \sum_{i, j}(\text { pixel int ensity }- \text { mean })^{2}$,

$\phi \in\left\{\phi_{1}, \phi_{2}, \phi_{3}, \phi_{4}\right\}$ : Out of eight, select four dominant orientations which contains higher energy distribution,

$i=1,2, \ldots 8$ and $j=1,2, \ldots 16$.

In this way, a 4-D feature map corresponding to the four filtered images for each shoeprint image is obtained. Figure 2 shows shoeprint image convolved with Gabor filter in eight directions. Out of these only four Gabor filtered images containing significant energy distribution have been considered as dominant to compute the variance feature map. Figure 3 shows the four dominant variance feature map for shoeprint image. It is seen that two different shoeprints posses different textural features. Finally, this feature map has been used to represent and match a query shoeprint image.

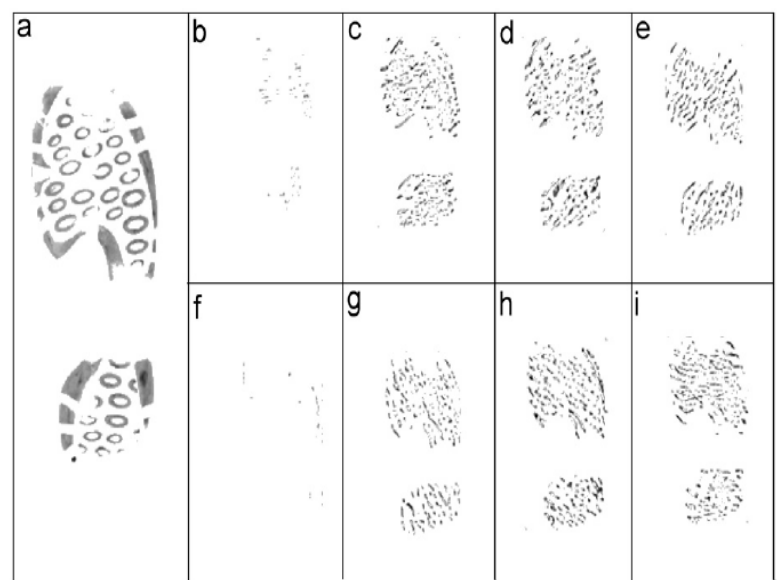

Fig.2. (a) Shoeprint image and (b)-1 Gabor filter bank in eight Orientations $\left(0^{0}, 22.5^{0}, 45^{0}, 67.5^{0}, 90^{0}, 112.5^{0}, 135^{0}\right.$, and $157.5^{\circ}$ ).

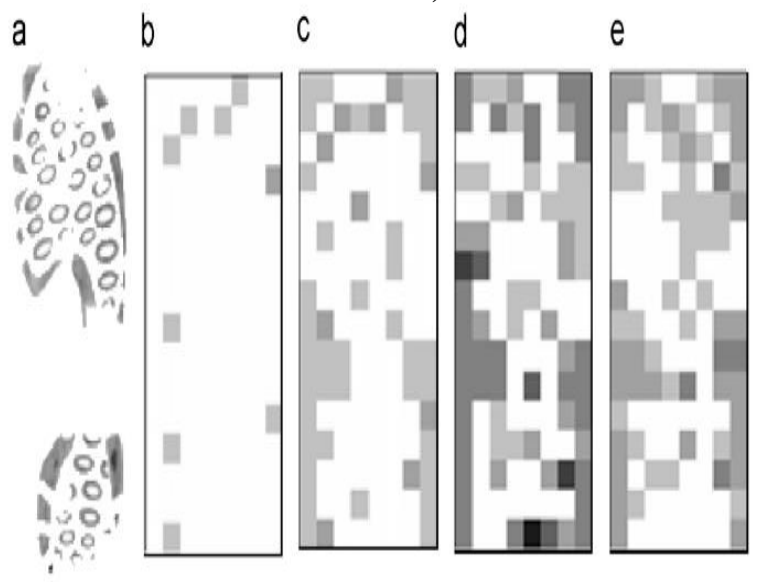

Fig. 3. (a) A typical example of shoeprint image and (b)-(e) four dominant variance features.

\section{DATABASE USED}

MATLAB is used for implementing and testing of the algorithm.

\subsection{Shoeprint Database}

Forensic analysis requires comparison of reference image against specific databases. These databases include:

Forensic image databases often contain one or more subdatabases:

1. Images that are collected from the scene of crime; with this database it is possible to link cases to each other.

2. Images of marks that are collected from the suspect (e.g., shoe marks that are made with shoes of a suspect); with this database in combination with the database of images that are collected from the scene of crime, it is possible to link suspect with cases.

3. Reference images (e.g., shoe marks from shoes that are commercially available, that can be used to determine which brand and make of shoe a certain shoe mark is from). 


\subsection{Database Generation}

Shoeprint database has been generated by inviting participants to tread on an inkpad (only once) and then stamp on an 11.69 " x 8.27" paper. Further the shoeprint images were digitized using a flatbed scanner of 300 dpi resolution and 256 level gray-scale mapping. Minimum two prints from each subject were acquired to ensure that, for each print there exists at least one matching print. All shoeprints belonging to the same pattern category has identical outsole patterns (ignoring the effects of shoe wear, shoe size, and image quality). The experiments had been conducted with a database consists of 40 shoeprint images. The shoeprint is converted from rgb jpeg format to grey scale image, before processing. The first shoeprint image of an individual was a full print without any rotation. The second database consists of shoeprint images with some rotation. These shoeprint images are used for testing shown in figure 4.

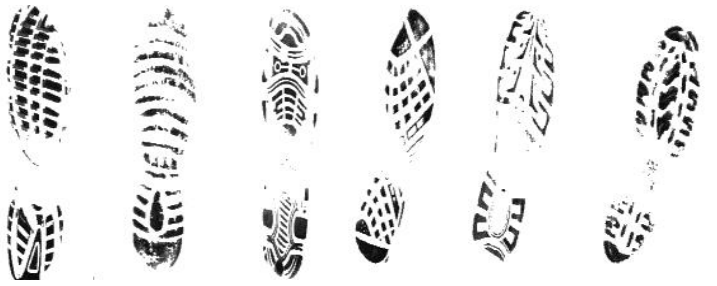

Fig.4 Database taken on paper, captured by webcam and then stored in database.

\section{RESULTS AND DISCUSSIONS}

Due to the freedom offered to the subjects, naturally the rotation, intensity variation (by means of contrast variation in the gray scale) or both have been introduced randomly in the test shoeprint images. The intensity variations introduced are because of variations in the pressure exerted by the subject and non uniformity in spreading of ink during image acquisition process. Intensity variations in the shoeprint image affects on its average power. But the appearance of directional patterns in a shoeprint does not get affected significantly. The maximum rotation introduced by the subject in the shoeprint was found 60 degree. To make the algorithm intensity invariant, the test shoeprint images have been normalized with respect to their mean in the beginning of the algorithm.

Experimentation for shoeprint matching has been carried for rotation, intensity variation and mix (both rotation and intensity variation) attacks on full print images. In this work two databases are used, one database consists of 40 straight shoeprint images and second database consists of 40 tilted shoeprint images. Two different methods, online and offline are used for shoeprint matching on these two databases. Therefore, total four methods have been used for shoeprint matching that is online method for straight and tilted images and offline method for straight and tilted images. This shoeprint image is taken on A4 size paper by pressing the shoe who's sole is painted with ink. In this online shoeprint matching, image is captured by webcam(10x digital zoom) and is given for recognition it is tallied by the stored database (trainee images) and the minimum value between the enrollment image and trainee is searched and the person with whom it matches by minimum distance it is assigned to that person and match is found. This method is applicable for both straight as well as tilted images. In offline shoeprint matching; image is taken from already stored database which consists of both straight and tilted shoeprint images. Also, in offline tilt and online tilt method, tilt query images are made straight then matching is done.

The result table 1 for shoeprint matching is shown below. Here the result is taken on 40 samples of shoeprint by online and offline methods for straight and tilted with some angle each.

In table no. 2 and 3 the result is shown of offline straight method and offline tilt method with matching parameters average variance, Euclidian distance, and angle for 40 shoeprint images each stored in database.

The outputs have been shown in GUI window as shown in figure 5 and figure 6 , developed with MATLAB image processing tool box.

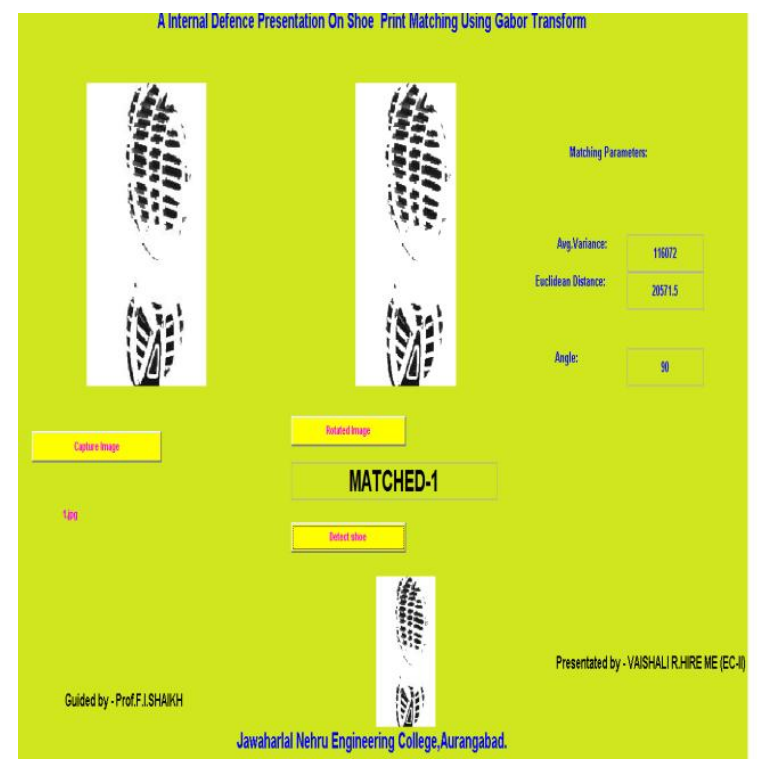

Fig.5 Offline and Online Straight Method GUI.

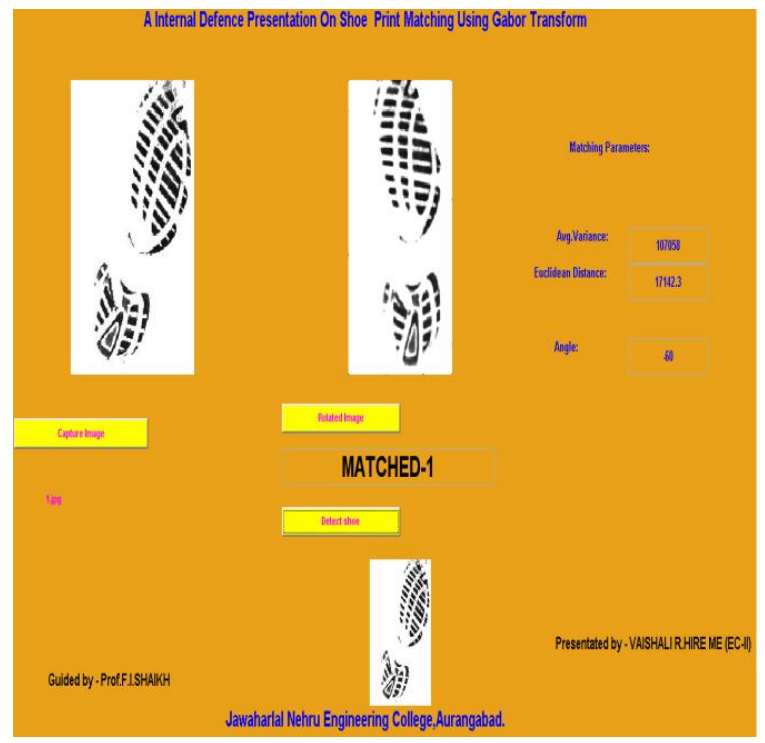

Fig.6 Offline and Online Tilt Method GUI.

This GUI contains three pop up window to capture, rotate, detect image, three image windows to show captured, rotated and detected shoeprint images, and four edit window to show matched person image number and matching parameters. 
Table 1. Results of all methods

\begin{tabular}{|c|c|c|c|c|c|}
\hline $\begin{array}{c}\text { Method } \\
\text { used }\end{array}$ & $\begin{array}{c}\text { Database } \\
\text { used }\end{array}$ & $\begin{array}{c}\text { No. of } \\
\text { images } \\
\text { In } \\
\text { database }\end{array}$ & $\begin{array}{c}\text { No. of } \\
\text { images } \\
\text { matched }\end{array}$ & $\begin{array}{c}\text { No. of } \\
\text { images } \\
\text { not } \\
\text { matched }\end{array}$ & $\begin{array}{l}\text { Nos. of images } \\
\text { not matched }\end{array}$ \\
\hline Offline & Straight & 40 & 37 & 03 & $20,21,25$ \\
\hline Offline & tilt & 40 & 37 & 03 & $8,19,22$ \\
\hline Online & Straight & 40 & 30 & 10 & $\begin{array}{c}3,25,33,4,6,14 \\
12,11,7,16\end{array}$ \\
\hline Online & tilt & 40 & 21 & 19 & $\begin{array}{c}40,16 \\
23,6,26,33,3,12,28 \\
35,2,38,36,39,31,30 \\
, 22,24,19\end{array}$ \\
\hline
\end{tabular}

Results

Offline Straight

Offline Tilt

Online Straight

Online Tilt

\section{CONCLUSIONS}

This work presents a technique for automatic shoeprint matching using multi resolution Gabor feature map. Shoeprint impressions can be input into the system using a digital scanner or a digital camera. During matching, a direction (orientation) of query shoeprint image has been estimated using Radon transform. Gabor transform has been used to extract genuine textural features in a shoeprint image. The matching score has been computed on the basis of Euclidian distance metrics. The technique is invariant to variations in intensity and rotation. The technique performs better compared to results obtained using PSD features for full print images with the rotation, intensity and mixed attacks at all the ranks. The matching accuracy is very good that is $90 \%$ for both offline straight and offline tilt matching method and the accuracy of online matching method is somewhat less as

$$
\begin{aligned}
& =90 \% \\
& =90 \% \\
& =75 \% \\
& =50 \%
\end{aligned}
$$

compared to offline matching method. The accuracy of online straight matching method is $75 \%$ and of online tilt matching method is $50 \%$. The simplified data entry of this system makes it a lower cost system to develop and maintain, as well as offering clients significant personnel cost savings. The implemented algorithm has an upper hand especially for partial prints. Performance of the algorithm has been evaluated in terms of percentage accuracy for full shoeprints. The method is robust to Gaussian white noise, and salt-pepper noise. Also, the work could be extended to evaluate the robustness of the algorithm to variations in scale and position of the shoe print image using orientation field or artificial neural network $[12,13]$ with a larger database available with forensic laboratories. A fast and accurate matching or indexing strategy is needed for a large shoeprint image 
database. There is continuing scope for more advanced local feature detectors and descriptors. New fast processors will be available to implement complicated algorithms, the technique of shoeprint recognition will be more fast, accurate and cheap.

\section{REFERENCES}

[1] W.J. Bodziak, Footwear Impression Evidence Detection, Recovery and Examination, second ed. CRC Press, 2000.

[2] A. Girod, "Presentation at the European Meeting for Shoeprint/Toolmark Examiners," 1997.

[3] A. Girod, "Computer Classification of the Shoeprint of Burglar Soles,” Forensic Science Int'1, vol. 82, pp. 59-65, 1996.

[4] N. Sawyer, 'SHOE-FIT A Computerized Shoe Print Database," Proc. European Convention on Security and Detection, pp. 86-89, May 1995.

[5] W. Ashley, "What Shoe Was That? The Use of Computerized Image Database to Assist in Identification," Forensic Science Int'l, vol. 82, pp. 67-79, 1996.

[6] A. Girod, "Presentation at the European Meeting for Shoeprint / Tool mark Examiners," 1997.

[7] A.Bouridane, A.Alexander, M.Nibouche, D.Crooks, Application of fractals to the detection and classification of shoeprints, in: Proceedings of the IEEE Conference on Image Processing,IEEE,0-7803-6297,2000,pp.474-477.

[8] G.Alexander, Computerized classification of the shoeprints of burglars soles, Forensic Science International 82 (1996) 59-65.

[9] A.Alexander, A.Bouridane, D.Crooks, Automatic classification and recognition of shoeprints, in: Proceedings of the Seventh International IEEE Conference on Image Processing and its Applications, vol.2, 1999, pp.638-641.

10] Z.Geradts, The image database REBEZO for shoeprints with developments on automatic classification of shoe outsole design, Forensic Science International 82 (1996)21-31.

[11] P.Toft, The Radon transforms - theory and implementation, Ph.D. Thesis, Department of Mathematical Modeling, Technical University of Denmark, 1996, pp. 6-7.

[12] J.G.Daugman, Two-dimensional spectral analysis of cortical receptive field profiles, Vision Research 20 (1980) 847-856.

[13] P. Chazal, J. Flynn, R.B. Reilly, Automated processing of shoeprint images based on the Fourier transform for use in forensic science, IEEE Transactions on Pattern Analysis and Machine Intelligence 27 (3) (2005) 1-10.

\section{Table number 2 and 3 are shown on next}

page. 
Table No. 2 Results of offline straight method with matching parameters

\begin{tabular}{|c|c|c|c|c|}
\hline \multicolumn{5}{|c|}{ Offline Straight Method } \\
\hline & \multicolumn{3}{|c|}{ Matching Parameters } & \multirow[b]{2}{*}{$\begin{array}{l}\text { Match } \\
\text { Y/N }\end{array}$} \\
\hline $\begin{array}{l}\text { Image } \\
\text { No. }\end{array}$ & $\begin{array}{l}\text { Ave. } \\
\text { Variance }\end{array}$ & $\begin{array}{l}\text { Eud. } \\
\text { Dist. }\end{array}$ & Angle & \\
\hline 1 & 116072 & 37275.6 & 90 & $\mathrm{Y}$ \\
\hline 2 & 106444 & 6284.99 & 80 & Y \\
\hline 3 & 116072 & 18414.7 & 90 & $\mathrm{Y}$ \\
\hline 4 & 106444 & 8329.61 & 80 & $\mathrm{Y}$ \\
\hline 5 & 116072 & 22752.2 & 90 & $\mathrm{Y}$ \\
\hline 6 & 106444 & 8533.47 & 80 & $\mathrm{Y}$ \\
\hline 7 & 116072 & 17497.7 & 90 & $\mathrm{Y}$ \\
\hline 8 & 106981 & 19563.5 & -80 & $\mathrm{Y}$ \\
\hline 9 & 116072 & 28057.6 & 90 & $\mathrm{Y}$ \\
\hline 10 & 106981 & 17407.1 & -80 & $\mathrm{Y}$ \\
\hline 11 & 106444 & 8405.72 & 80 & $\mathrm{Y}$ \\
\hline 12 & 106444 & 10953.8 & 80 & $\mathrm{Y}$ \\
\hline 13 & 116072 & 11831.4 & 90 & $\mathrm{Y}$ \\
\hline 14 & 116072 & 9265.16 & 90 & $\mathrm{Y}$ \\
\hline 15 & 116072 & 20519.5 & 90 & $\mathrm{Y}$ \\
\hline 16 & 111632 & 1534.04 & 70 & $\mathrm{Y}$ \\
\hline 17 & 116072 & 13840.8 & 90 & $\mathrm{Y}$ \\
\hline 18 & 116072 & 15745.3 & 90 & $\mathrm{Y}$ \\
\hline 19 & 106444 & 9625.7 & 80 & $\mathrm{Y}$ \\
\hline 20 & 116072 & 61330.4 & 90 & $\mathrm{~N}$ \\
\hline 21 & 106981 & 45073.2 & -80 & $\mathrm{~N}$ \\
\hline 22 & 116072 & 15980.2 & 90 & $\mathrm{Y}$ \\
\hline 23 & 106981 & 13239.2 & -80 & $\mathrm{Y}$ \\
\hline 24 & 106981 & 14185.5 & -80 & $\mathrm{Y}$ \\
\hline 25 & 106981 & 53847.6 & -80 & $\mathrm{~N}$ \\
\hline 26 & 106981 & 11078.8 & -80 & $\mathrm{Y}$ \\
\hline 27 & 106444 & 12375.1 & 80 & $\mathrm{Y}$ \\
\hline 28 & 106444 & 9010.77 & 80 & $\mathrm{Y}$ \\
\hline 29 & 116072 & 18867.1 & 90 & $\mathrm{Y}$ \\
\hline 30 & 116072 & 9878.58 & 90 & $\mathrm{Y}$ \\
\hline 31 & 116072 & 17646 & 90 & $\mathrm{Y}$ \\
\hline 32 & 106444 & 5798.92 & 80 & $\mathrm{Y}$ \\
\hline 33 & 106444 & 2537.12 & 80 & $\mathrm{Y}$ \\
\hline 34 & 106981 & 10990.9 & -80 & $\mathrm{Y}$ \\
\hline 35 & 116072 & 5877.19 & 90 & $\mathrm{Y}$ \\
\hline 36 & 106444 & 5040.37 & 80 & $\mathrm{Y}$ \\
\hline 37 & 116072 & 30520.9 & 90 & $\mathrm{Y}$ \\
\hline 38 & 116072 & 24147.8 & 90 & $\mathrm{Y}$ \\
\hline 39 & 106981 & 12016.3 & -80 & $\mathrm{Y}$ \\
\hline 40 & 111632 & 7255.36 & 70 & $\mathrm{Y}$ \\
\hline
\end{tabular}

Table No. 3 Results of offline tilt method With matching parameters

\begin{tabular}{|c|c|c|c|c|}
\hline \multicolumn{5}{|c|}{ Offline Tilt Method } \\
\hline \multicolumn{4}{|c|}{ Matching Parameters } & \multirow[b]{2}{*}{$\begin{array}{l}\text { Match } \\
\text { Y/N }\end{array}$} \\
\hline $\begin{array}{l}\text { Image } \\
\text { No. }\end{array}$ & $\begin{array}{l}\text { Ave. } \\
\text { Variance }\end{array}$ & $\begin{array}{l}\text { Eud. } \\
\text { Dist. }\end{array}$ & Angle & \\
\hline 1 & 106444 & 28548.3 & -60 & $\mathrm{Y}$ \\
\hline 2 & 106444 & 10076.4 & -70 & $\mathrm{Y}$ \\
\hline 3 & 106444 & 12280.5 & -70 & $\mathrm{Y}$ \\
\hline 4 & 106444 & 16657 & -70 & $\mathrm{Y}$ \\
\hline 5 & 106444 & 9218.68 & -60 & $\mathrm{Y}$ \\
\hline 6 & 106981 & 13725.6 & -80 & $\mathrm{Y}$ \\
\hline 7 & 106444 & 6953.65 & -70 & $\mathrm{Y}$ \\
\hline 8 & 106444 & 41079.5 & -60 & $\mathrm{~N}$ \\
\hline 9 & 116072 & 21551.8 & -90 & $\mathrm{Y}$ \\
\hline 10 & 106444 & 18119.3 & -70 & $\mathrm{Y}$ \\
\hline 11 & 106444 & 17304.5 & -70 & $\mathrm{Y}$ \\
\hline 12 & 106444 & 41092.7 & -70 & $\mathrm{Y}$ \\
\hline 13 & 106981 & 10115.2 & -80 & $\mathrm{Y}$ \\
\hline 14 & 106444 & 7549.54 & -70 & $\mathrm{Y}$ \\
\hline 15 & 106444 & 18983.7 & -70 & $\mathrm{Y}$ \\
\hline 16 & 106981 & 12529 & -80 & $\mathrm{Y}$ \\
\hline 17 & 106444 & 5755.81 & -60 & $\mathrm{Y}$ \\
\hline 18 & 106444 & 10370.7 & -70 & $\mathrm{Y}$ \\
\hline 19 & 106444 & 39247.8 & -70 & $\mathrm{~N}$ \\
\hline 20 & 106444 & 21615.4 & -60 & $\mathrm{Y}$ \\
\hline 21 & 106444 & 22137.5 & -70 & $\mathrm{Y}$ \\
\hline 22 & 106444 & 17365 & -80 & $\mathrm{~N}$ \\
\hline 23 & 106444 & 11647.7 & -70 & $\mathrm{Y}$ \\
\hline 24 & 106444 & 12480.8 & -60 & $\mathrm{Y}$ \\
\hline 25 & 106444 & 18948.6 & -70 & $\mathrm{Y}$ \\
\hline 26 & 106444 & 10015.5 & -70 & $\mathrm{Y}$ \\
\hline 27 & 106444 & 21536.3 & -70 & $\mathrm{Y}$ \\
\hline 28 & 106444 & 12419.8 & -70 & $\mathrm{Y}$ \\
\hline 29 & 106444 & 21624.8 & -70 & $\mathrm{Y}$ \\
\hline 30 & 106444 & 5270.28 & -70 & $\mathrm{Y}$ \\
\hline 31 & 106444 & 16617.6 & -70 & $\mathrm{Y}$ \\
\hline 32 & 106444 & 11531.4 & -70 & $\mathrm{Y}$ \\
\hline 33 & 106444 & 7767.33 & -70 & $\mathrm{Y}$ \\
\hline 34 & 106981 & 16354.2 & -80 & $\mathrm{Y}$ \\
\hline 35 & 106444 & 2656.13 & -70 & $\mathrm{Y}$ \\
\hline 36 & 106981 & 12615 & -80 & $\mathrm{Y}$ \\
\hline 37 & 106444 & 51568.2 & -60 & $\mathrm{Y}$ \\
\hline 38 & 106444 & 16120.2 & -60 & $\mathrm{Y}$ \\
\hline 39 & 106813 & 18941.2 & -50 & $\mathrm{Y}$ \\
\hline 40 & 106444 & 10085.7 & 20 & $\mathrm{Y}$ \\
\hline
\end{tabular}

